\title{
Building Portraits
}

THEORY

\section{Elena Manferdini}

\begin{abstract}
Building Portraits is a suite of elevation studies developed by Atelier Manferdini for an exhibition at the Art Institute of Chicago in 2015 called Building the Picture and a subsequent solo show at Industry Gallery in Los Angeles in 2016 called Building Portraits. These 42 drawings were produced during the past two years and they explore the potential of intricate scripted line work depicting building facades. The collection exists simultaneously as architectural research and as autonomous artwork. These drawings can be understood as scaled down reproduction of buildings, and at the same time as full scale printed artifacts. The collection plays with the graphic potentials of woven grids and scripted vector lines, while exploring the canonical relationships of shape vs form, ground vs figure, pattern vs coloration, orientation vs posture. The title of the suite Building Portraits alludes to two distinct disciplines, the field of architectural drawings, building, and the one of fine artistic pictures, portraits. This body of work tries to claim a territory where these two attitudes find a common ground, where pixels and vectors get closer in scale of perception.
\end{abstract}

Keywords: architectural drawings, scripted drawings, Mies van der Rohe, facades

Through the centuries, drawings have measured the level of cultural and technical knowledge reached by a society in its various historical moments. Drawings, like glass lenses, are a medium through which 
one can observe the way new tools and techniques are challenging the normative way we describe our surroundings. By nature, graphic representation is mutable and resistant to strict codifications; drawings have the ability to change in scale, style, form, accuracy, pictorial and technical resonance. They easily jump from field to field, often acquiring new potentials or losing their original meaning. They can be armatures for geometrical descriptions or mere vessels of communication.

If we would take on the task to trace the lineage of the "architectural drawing," we would quickly be confronted by a difficult dichotomy. Architectural drawings are the product of an inherent duality: they can be imaginative or deterministic, speculative or technical, general or prescriptive. Regardless of these dualities, they still possess the ability to be called "architectural drawings." This moniker to falls within a wide spectrum of possible representational expressions that are not easy to categorize. They can be the manifestation of the architect's artistic ability, and at the same time a demonstration of his/her technical savoir-faire.

The reason for this ambiguity lies in the scale of architecture. The medium of architecture is not a drawing, but a fully built object - usually larger than its form of graphic representation. Therefore the working space of a drawing is a disciplinary playground, where architects create forms of memory, and not the work itself. This scalar documentation (bi or three dimensional) can manifest itself as a set of instructions on how to build in the real architectural medium, or intuitions of what the fully present architectural object could look like.

Drawings are the quintessential visualization of the tension between foresight and reflection, between concreteness and abstraction. In other words, they oscillate between being a way to imagine a plausible reality, or simply a means to an end. Because they are far enough from the real medium of architecture, they are free from physical inherent limitations.

The risk associated with working through graphic techniques (rather than the architectural medium) is that architectural drawings inherently may become hostages of other disciplines: fine art, on one side of the spectrum, and commercial representation, on the other. In this already difficult equation, digital tools have introduced another layer of complexity and an array of unprecedented possibilities to imagine this ever-changing territory. For instance, the realm of the architectural medium unexpectedly entered the abstraction of the architectural drawing with the fast advancement of digital photorealistic representation, three dimensional data collection, and material simulation.

This research is not an attempt to find a new overall post-digital diagram able to explain the faceted nature of our updated drawing skill-set. In fact, despite the various heroic attempts to draw it, this 


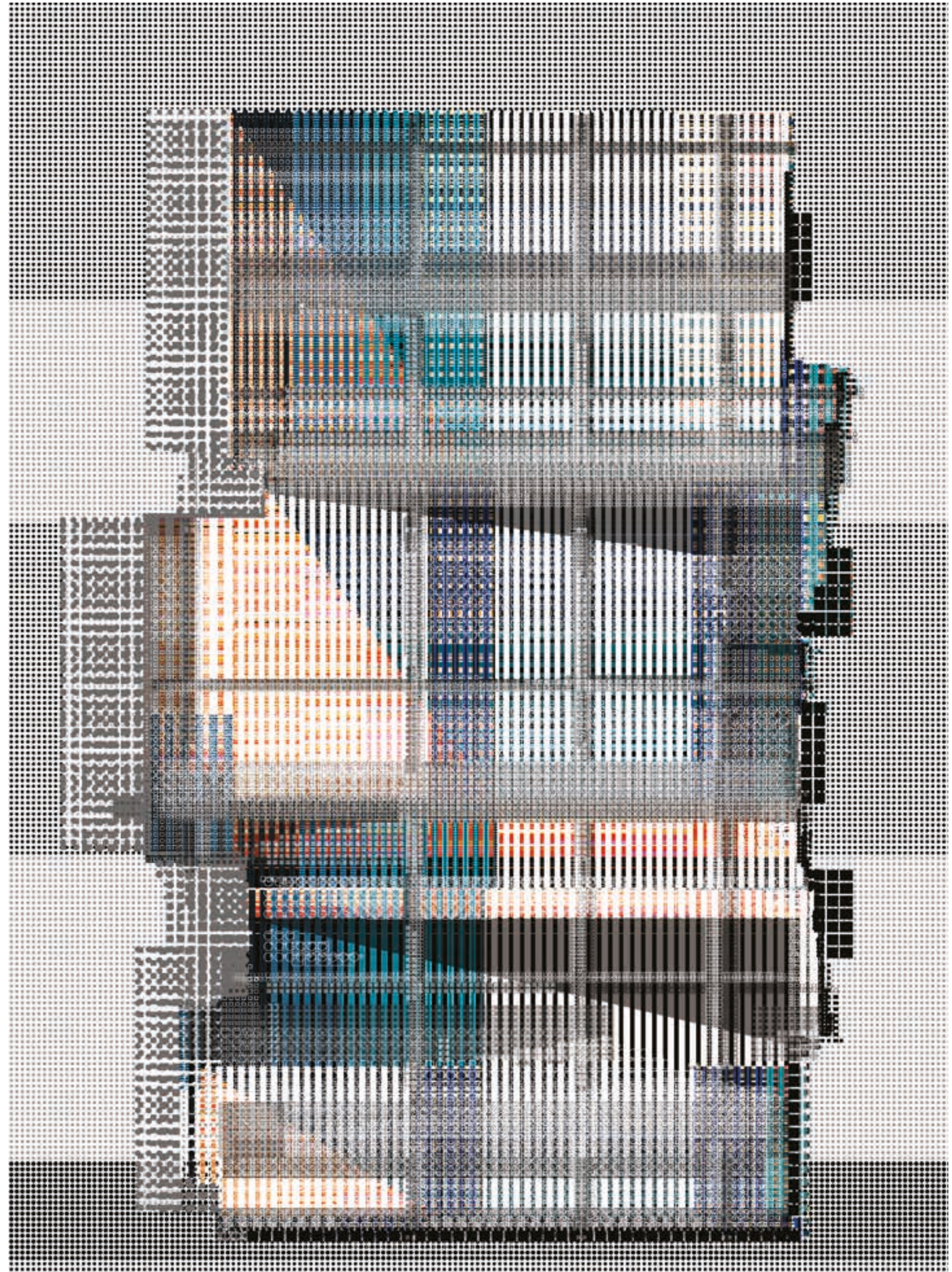

Figure 1. Building Portraits V. Series of Four Drawings 2015. Archival Ink Prints.

San Francisco Museum of Modern Art permanent collection. 
diagram might simply not exist, or might be too simplistic to matter as a valid thinking tool. Instead this research revolves around a specific relationship between digital scripted drawings and analogue pictures of the architectural medium. The hope behind choosing these two points of departure is to work out ways in which an analog architectural medium - a picture of a real building - can enter the abstract space of the architectural drawing. This body of work makes a case that scripted drawings have now the ability to carry an enormous amount of data and therefore we are closing the gap between the analog medium of architecture and drawings.

To prove this hypothesis, the initial research used as a point of departure a well-known analog picture of Ludwig Mies van der Rohe's Lake Shore Drive apartments building facade (Chicago, 1958-51). The procedure was to take the original black and white photograph of the architect's masterpiece, scan it, script it with discrete vector lines and weave it with a second colorful one authored by Atelier Manferdini, at a different scale of resolution. A series of cyclical operations were applied to the raster image in order to transform the image into a set of vector lines (Fig. 1).

The decision to start a set of autonomous drawings on patterns and facades came from a personal fascination with the work of Mies van der Rohe and the aesthetic value of his elevations. His work represents a perfect case-study to explore how scripted line work and ordered mullions could inform each other, and therefore provides ways to work with the medium of architecture - mullions - in the drawing spacegrids. The selection of Mies' buildings was guided also by the timid attempt to link meaning to geometry. Mies' constructions are historically famous examples of how the geometrical ordering system of the grid embodied a political meaning in our discipline. Mies' elevations, like many other Modernist works, projected an egalitarian image of the built environment. At a time when the reading of a modern building was supposed to be a by-product of its constructive technology, the modularity of its assembly system was asked to reinforce the idea of technical efficiency and social democracy.

Following this tradition, Mies' buildings assumed the generic attributes of production: for instance the facade adopted ready-made steel members as mullion systems; his elevations revealed the loadbearing structure and its construction methods became their primary expression. If one, though, takes a closer look at one of his facades, it is possible to discover that he often corrupted the functionality of his envelopes with ornamental fine grain mullions that had no use other than creating an interesting optical effect. Furthermore the effect achieved by these black mute big buildings in the city was all but generic: the steel mullions, along with the dark glass curtain wall, were able to create vibrant reflections that dynamically multiplied the image of one building onto the surroundings ones. 


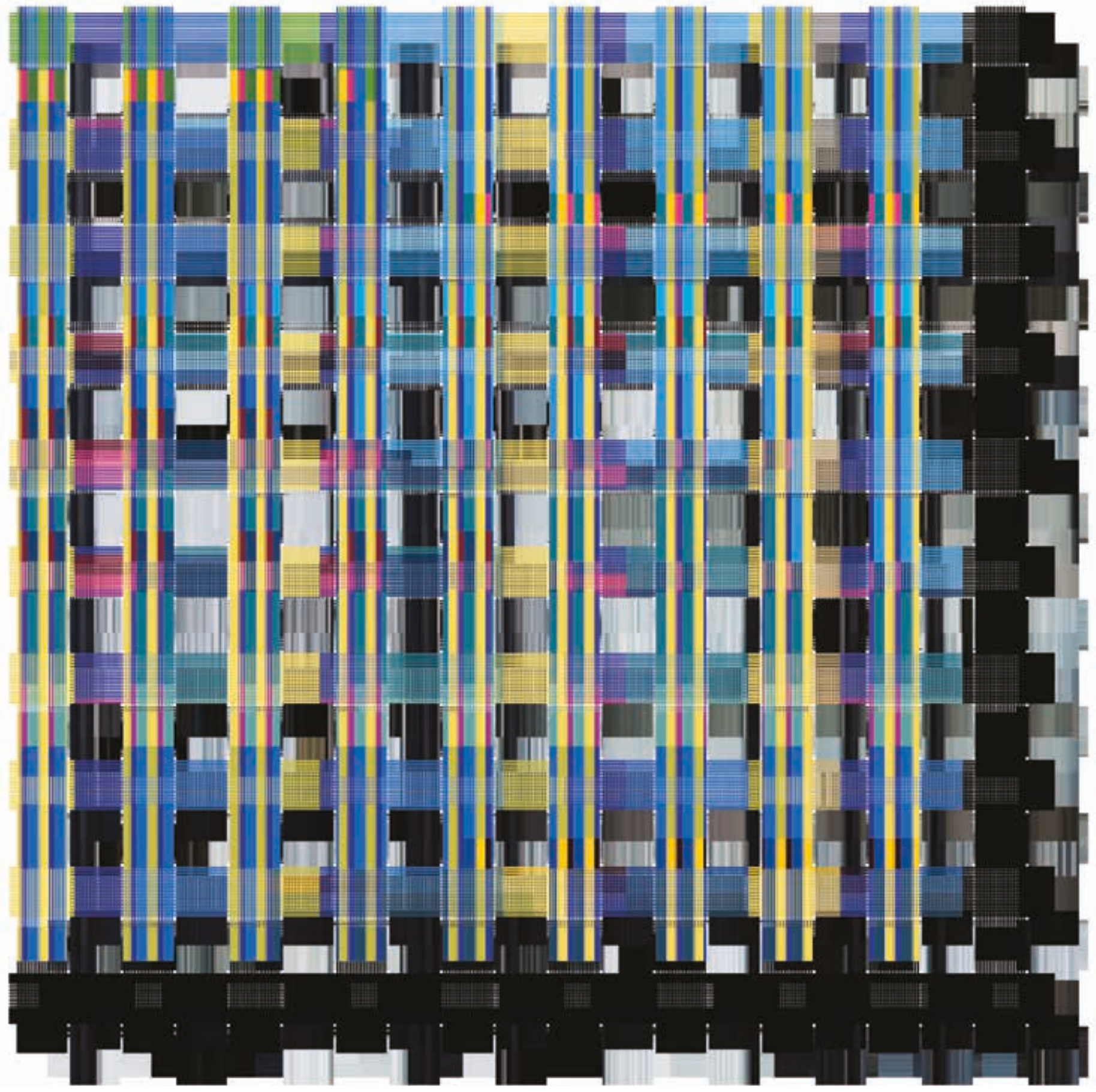

Figure 2. Building the Picture III, 2014. Print on Aluminum 24" x 24". 
Building upon Mies' facade and the discrepancies produced between modules and functionality, this first drawing (Fig. 2) subverts the mechanical use of the grid and its relationship to the modular systems in building construction. For instance the scale of the weave and fenestrations do not correspond to the location of the slabs behind them. The role of the grid in this drawing is the one of "picturing" ideas of fenestrations; often out of scale or with misaligned edges, the window pattern suggests a familiarity that at times reveals instead a radical departure from what we know as being generic.

Modular rigor is also applied to the chromatic finish of the facade: a relatively small selection of colors is able to create a wide variety of shades because of the intricate digital juxtapositions and overlay of treads next to each other. The computational process of overlaying pictures to drawings and finely weaving them together is able to merge the sterilizing line of the computer with the fertile line of city pictures. This is how computational abstraction and photorealistic figuration can be woven with each other in order to simultaneously occupy the viewers' perception. In addition to that, the weaves present a set of discrepancies and fringes when they reach the borders of the buildings: as they fray, they dismantle any canonical understanding of boundaries as symbols of hierarchy and traditional typology.

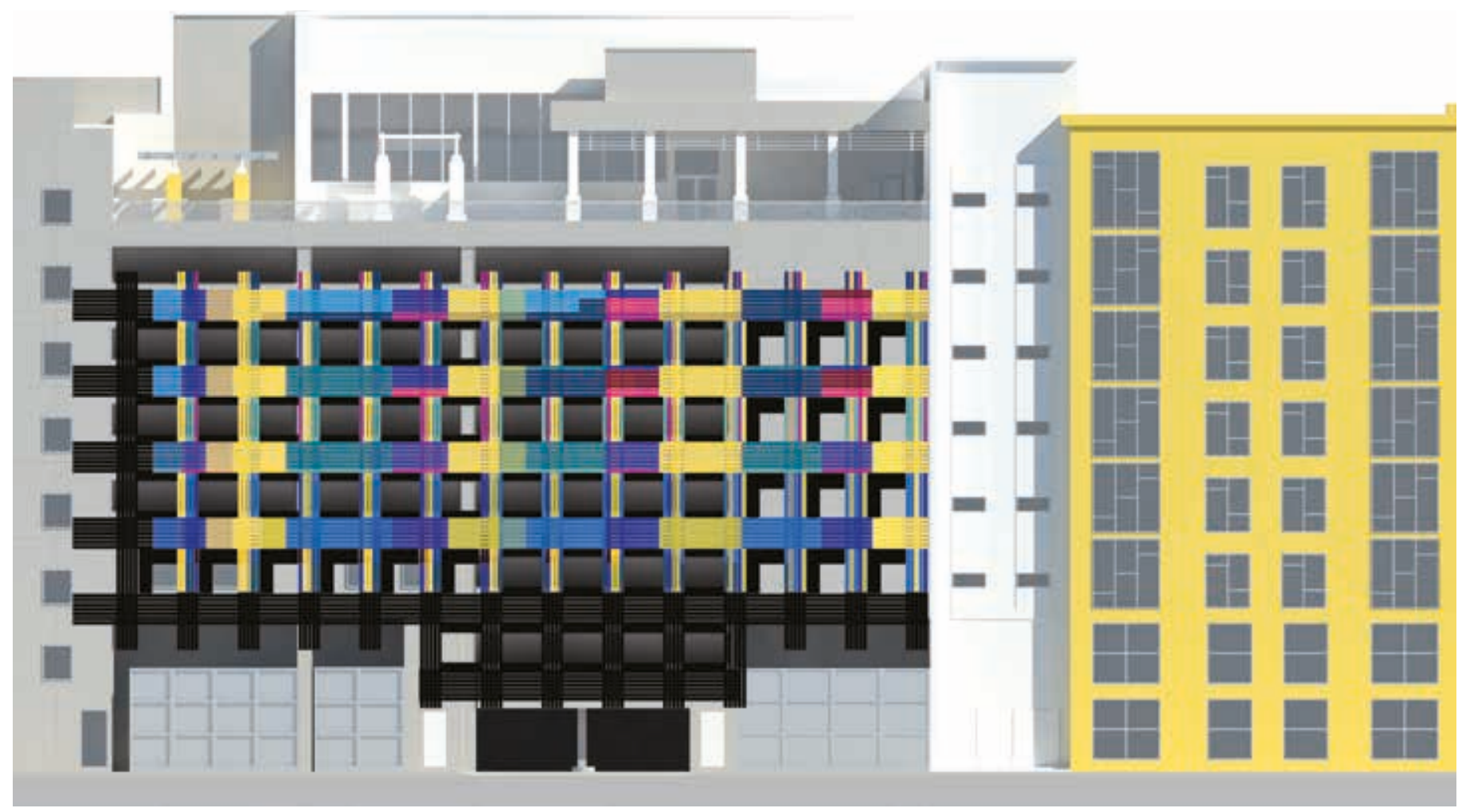

Figure 3. Hermitage Façade Drawing. Visualization of ongoing Façade for the Hermitage Garage in Saint Petersburg Florida 2016 

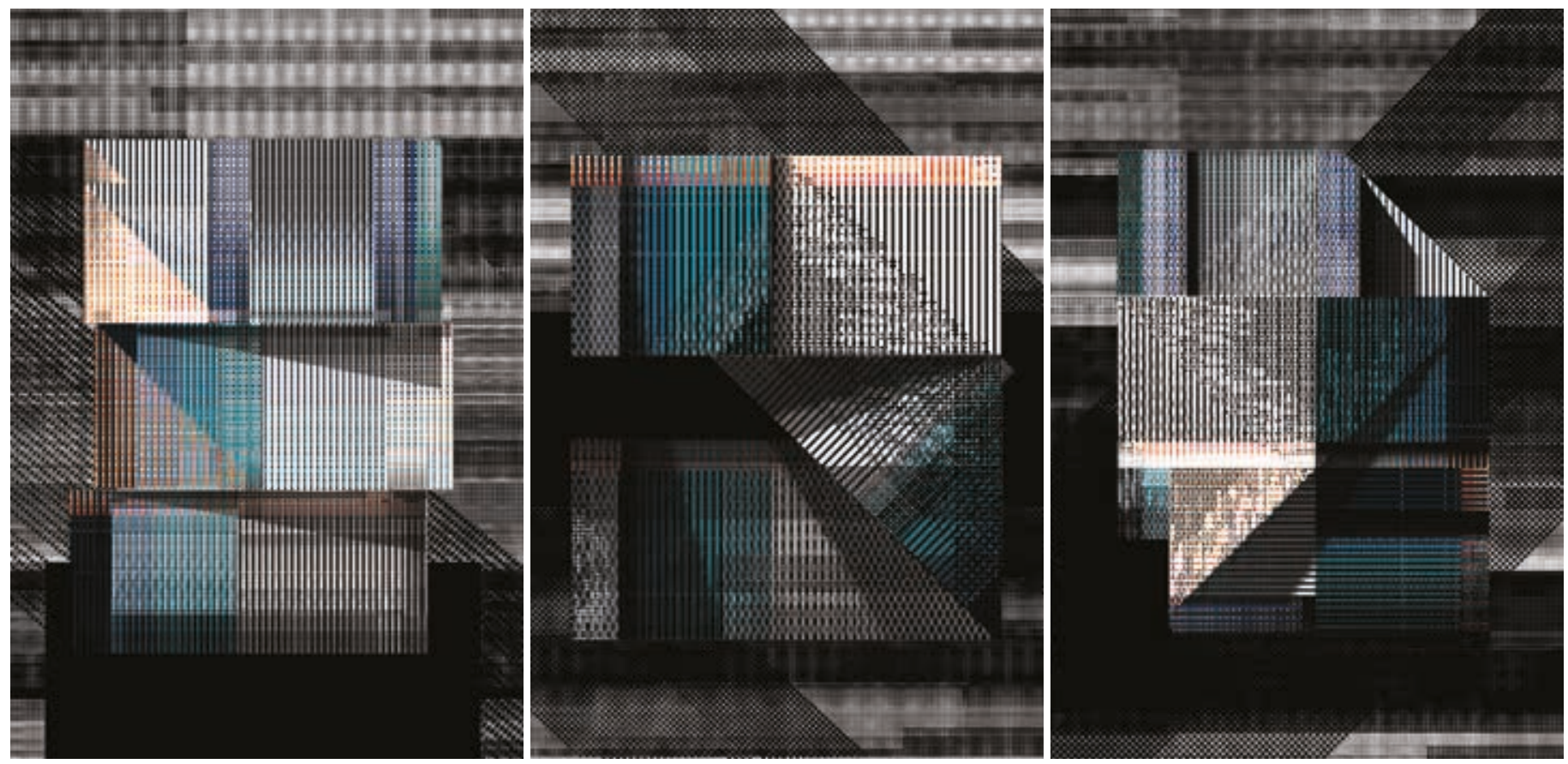

Figure 4. Forms and Ground I, Forms and Ground II, Forms and Ground III. Series of Three Drawings, 2015. Archival Ink Prints 13"x 19".

Building the Picture updates the project of the grid in the age of computation and globalization. The belief behind this research is that the grid applied to city facades (with a revised contemporary materiality and computational geometry) is able, once again, to estrange the buildings from the ordinary and render them unique architectural experiences.

To test these theories, an initial Building Portraits drawing (Fig. 3) has been the point of departure for the design of a full scale facade application in Florida. The drawing space has been scaled up to a 40' $x$ 80' architectural wall. The building facade is attached to a blank parking structure serving the newly constructed Hermitage housing project in the city of Saint Petersburg. Interestingly, parking facades are designed to a necessary degree: their appearance is often straightforward, earnest, and most important of all, non-exclusive. They are underestimated as anonymous. Nevertheless they also own a strong identity that inadvertently imposes itself (Walter Gropius called this "the unintentional beauty of industrial buildings"). These facades display a specific brut aesthetic that exists in architecture in the absence of routine human interaction.

The applied facade will introduce a new and contemporary sensibility to the structure through graphic, colors and shallow depth. Simultaneously, it will give a sense of human inhabitation to the parking garage.

If this initial suite of drawings for the exhibition Building the Picture at the Art Institute of Chicago originated from the desire to update 

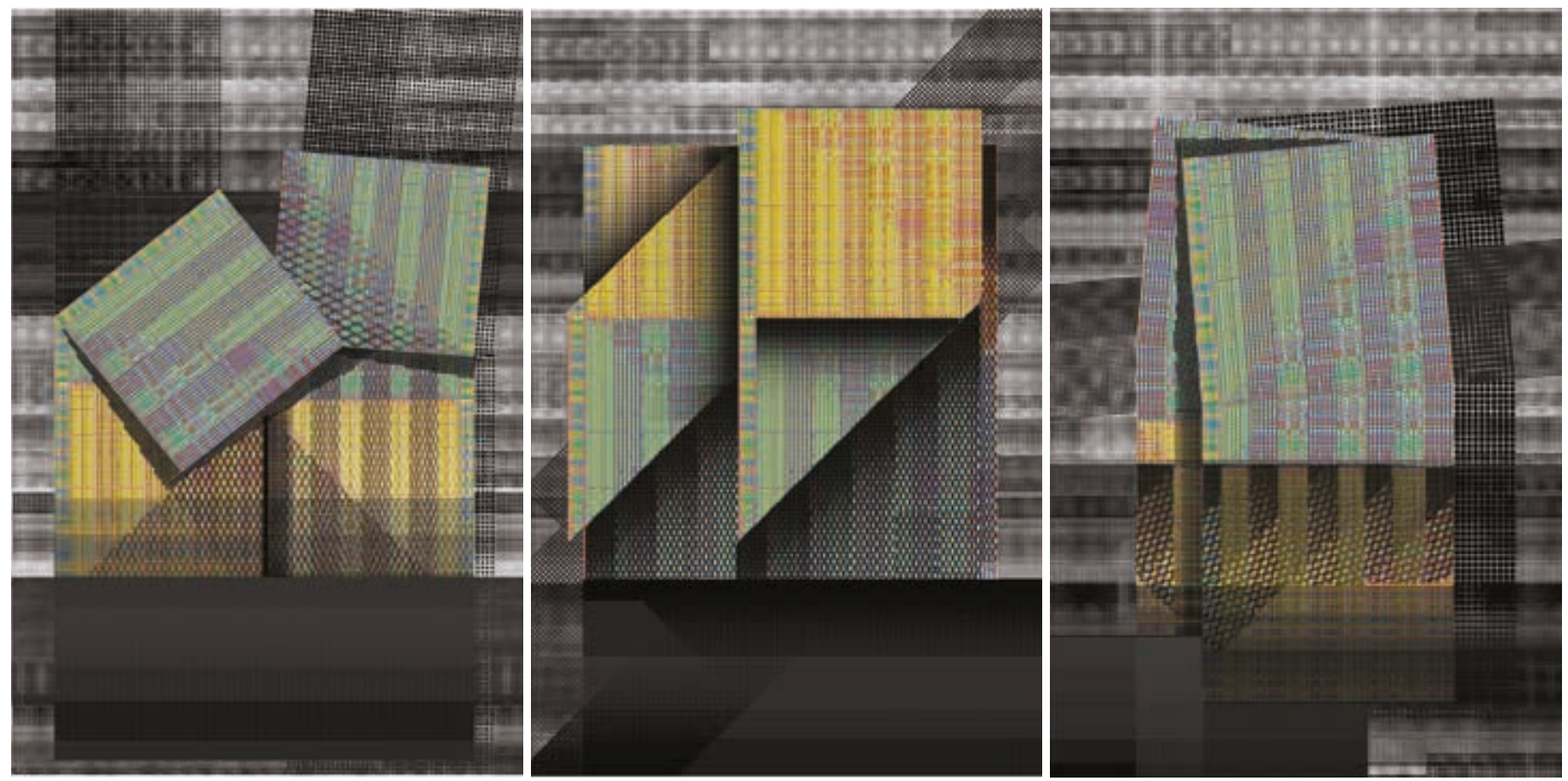

Figure 5. Postures and Ground I, Postures and Ground II, Postures and Ground III. Series of Three Drawings, 2015. Archival Ink Prints 13"x 19".
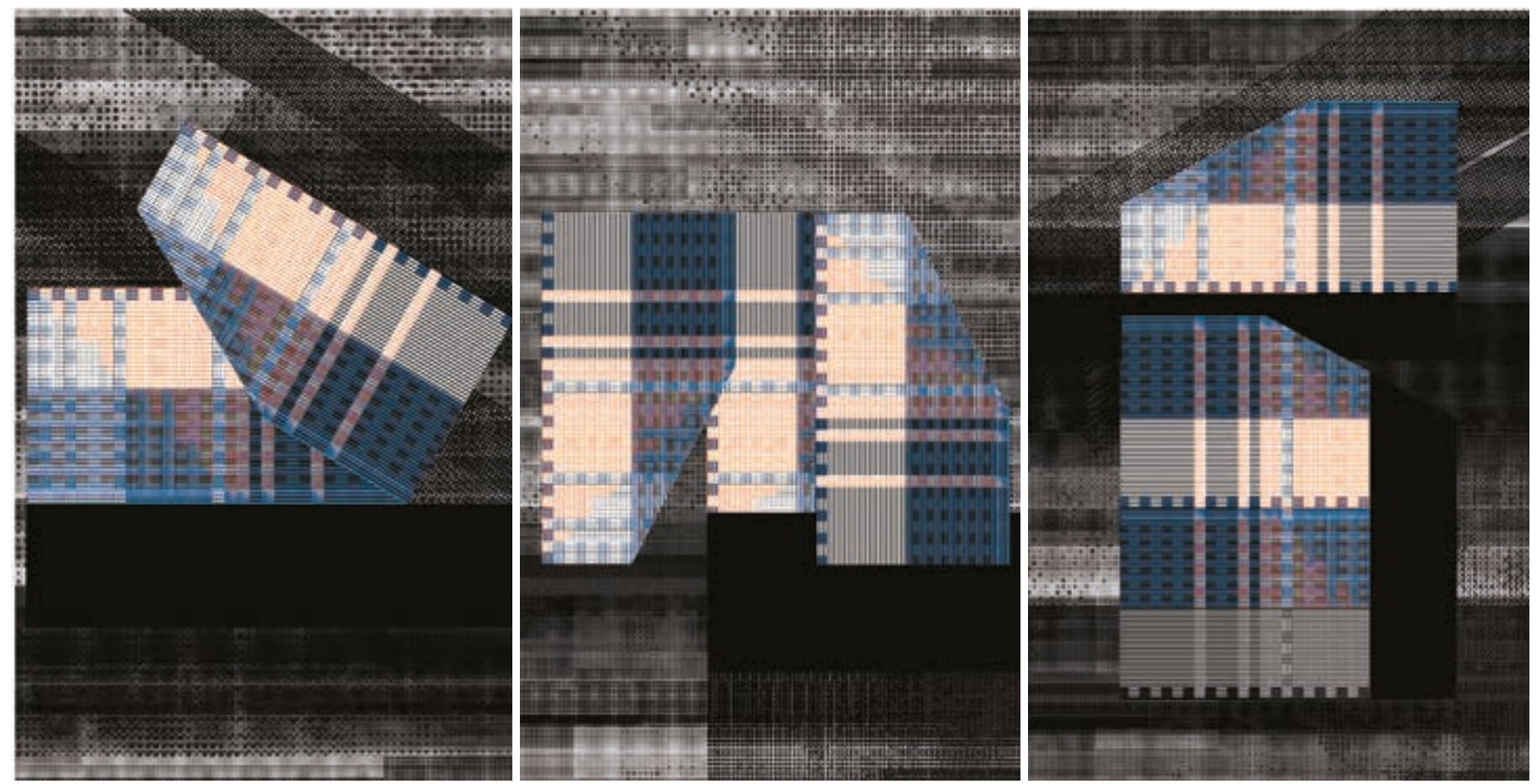

Figure 6. Postures and Ground IV, Postures and Ground V, Postures and Ground VI. Series of Three Drawings, 2015. 

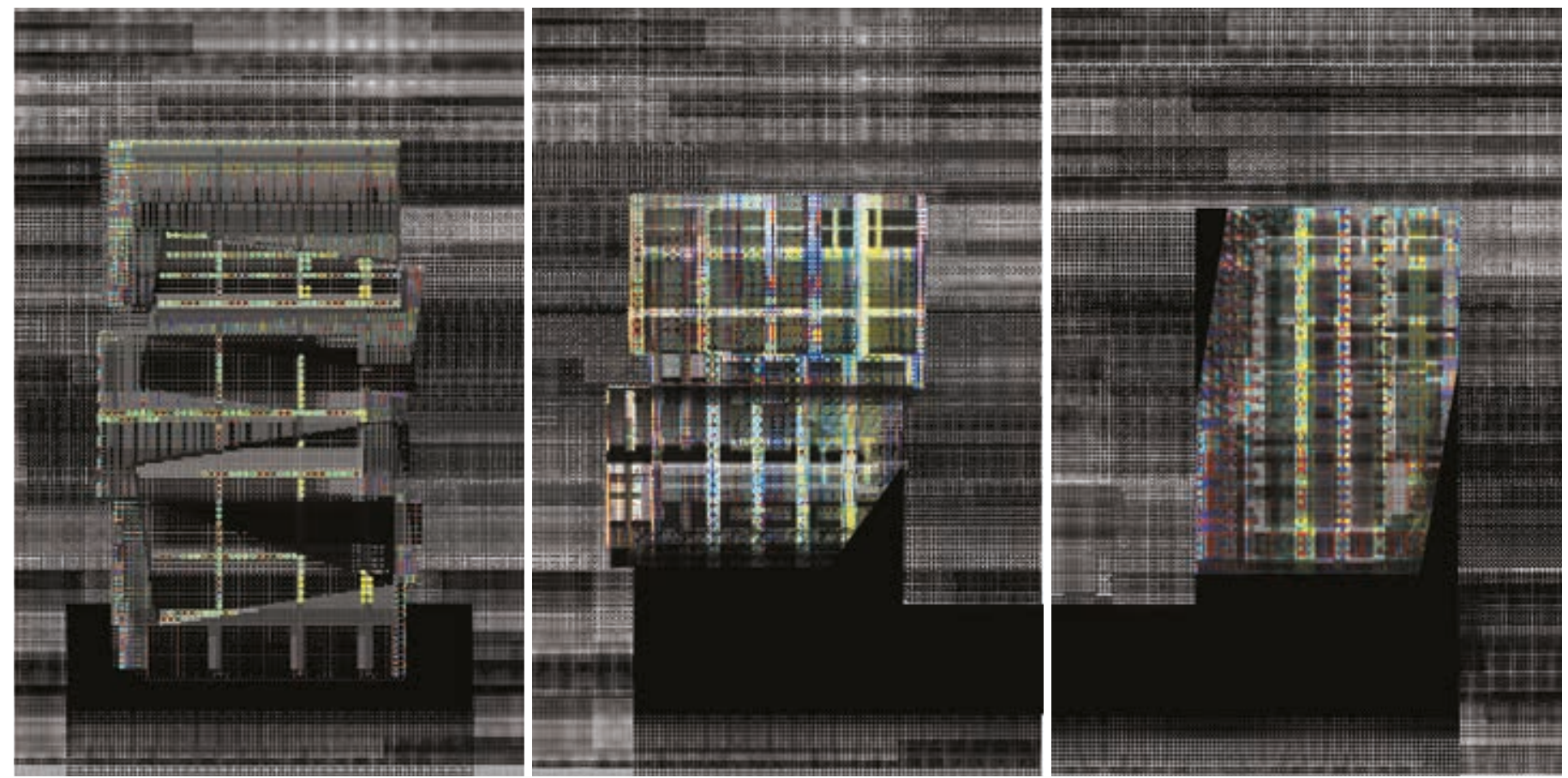

Figure 7. Forms and Ground_IV, Forms and Ground_V, Forms and Ground_VI. Series of Three Drawings, 2015. Print on Aluminum 24"x 36".

the relationship between drawings and architectural facades, in time the work evolved in three dimension and engaged with the canonical volumetric relationships of shape vs form, ground vs figure, pattern vs coloration, and orientation vs posture (Fig. 4).

The natural development of this research evolved in a second set of drawings, called Building Portraits, for a solo show at Industry Gallery in Los Angeles. This new body of work came from the realization that if Modern Architecture felt short to express the complexity of a current sensibility, where diversity and heterogeneity are the essence of contemporary culture, then a new set of three dimensional operations are nowadays needed to update the political project of architecture. If the introduction of the anomaly of the grid on a flat facade might not always be a sufficient tool to represent the multifaceted political subjectivity that is constantly reformulated in the age of globalization, then contemporary architecture needs also to develop original responses to the generic through a variety of massing strategies (Fig. 5).

This second suite of drawings tries to define such contemporary generic. These compositions argue for the fertile juxtaposition of simple shapes, uniquely oriented in relationship to the ground. The work explores orientation vs posture or shape vs form (Figs. 6-7). 
The conceptual basis for this entire research is rooted in the belief that contemporary architecture is now able to produce new geometrical forms that can no longer be structured on the traditional opposition between front and back, private and public, top and bottom. These new envelopes rely on highly choreographed experience of the surface, with its loose orientation on the ground. The work is not based on an historical-interpretive methodology or logical argumentation, but wants to provide a working methodology towards a contemporary aesthetic of computational, multi-directional, dynamic ordering system.

\section{Acknowledgements}

A previous, abridged version of this article was published as "Miesian Grids and the Domain of Ink" in Architectural Design "Mass Customized Cities," November 2015.

We thank John Wiley and Sons, publisher of Architectural Design, for their consent to the publication of this expanded version of the original article.

Elena Manferdini graduated in Civil Engineering from the University of Bologna (Bologna, It.) and later received her Master of Architecture and Urban Design from the University of California, Los Angeles. In 2004 she founded Atelier Manferdini in Venice CA. In 2011 she was one of the recipients of the prestigious annual grants from the United States Artists (USA) in the category of architecture and design. Since 2003 Elena has been part of the design faculty at the Southern California Institute of Architecture (SCl-Arc), where she is now the Graduate Programs Chair. E-mail: elena manferdini[AT]sciarc.edu 